\title{
AVALIAÇAOO DAS NECESSIDADES DOS SERVIÇOS OFICIAIS DE SAÚdE PÚBLICA PARA O MUNICAPIO DE SÃO PAULO. A MÉDIO E LONGO PRAZO
}

\author{
João YUNES (1) \\ Joaquim Francisco CARDOSO (2) \\ Mário Laranjeira de MENDONÇA (2)
}

Yunes, J.; Cardoso, J. F. \& Mendonça, M. L. de - Avaliação das necessidades dos serviços oficiais de saúde pública para o município de São Paulo, a médio e longo prazo. Rev. Saúde públ., S. Paulo,3(2):213-217, dez. 1969.

RESUMo - São apresentadas as necessidades de unidades sanitárias a médio e a longo prazo, correspondente aos respectivos anos de 1975 e 1990. Tal estudo integrou o Plano Urbanístico Básico de São Paulo (PUB) e as unidades propostas sempre obedeceram o critério de unidades integradas ou polivalentes. Para definição dos padrões propostos levou-se em conta o tamanho da população, densidade demográfica, tamanho da área, raio de atendimento e classe sócio-ećonômicä. As unidádes sanitárias foram divididas em três tipos e definidos, os seus programas de acôrdo com as respectivas atribuições propostas. A responsabilidade funcional foi definida para o setor público municipal, estadual e previdenciário.

\section{$1-$ I N T RODUCAO}

A avaliação das necessidades foi feita tendo em vista os equipamentos para os serviços oficiais de saúde pública, aqui representados pelas unidades sanitárias. $O$ seu dimensionamento refere-se ao Município de São Paulo e as necessidades serão projetadas para cada uma das áreas das unidades definidas pelo estudo de estrutura urbana do Plano Urbanístico Básico de São Paulo (PUB). Os padrões propostos serão a médio e a longo prazo, ou seja, em 1975 e 1990, respectivamente.

\section{2 - PADROES PROPOSTOS}

As unidades de saúde deverão sempre obedecer a critérios de integração física e funcional, em unidades polivalentes. Tôda a assistência médica oficial, preventiva e curativa, prestada pelos órgãos de saúde estadual e municipal deverá ser estabelecida em íntima coordenação, para evitar dispersão de esforços, dualidade de serviços e baixo rendimento quantitativo e qualitativo.

$\mathrm{Na}$ proposição dos novos padrões para os diferentes tipos de unidades sanitárias,

Recebido para publicação em 1-9-1969.

(1) Do Centro de Estudos de Dinâmica Populacional, anexo à Cadeira de Estatistica Aplicada a Saude Pública da Faculdade de Higiene e Saúde Pública da USP, São Paulo - Brasil; da Clínica Pediátrica da Faculdade de Medicina da USP, São Paulo - Brasil e da ASPLAN (Assessoria em Planejamento) - São Paulo, Brasil. (Coordenador do Setor Saúde do Plano Urbanistico Básico (PUB) de São Paulo).

(2) Da ASPLAN (Coordenador do Setor Social do PUB de São Paulo).

(3) Da ASPLAN (Diretor do PUB de São Paulo). 
YUNES, J.; CARDOSO, J. F. \& MENDONÇA, M. L. de - Avaliação das necessłdades dos serviços de saúde pública para o municipio.. Rev. Saúde públ., S. Paulo, 3(2):213-217, dez. 1969.

levou-se em conta o tamanho da população, densidade demográfica, tamanho da área, raio de atendimento e classe sócio-econômica.

As unidades sanitárias em nível local prestarão serviços executando programas de saúde pública em função das condições sanitárias da comunidade, através dos seus diferentes tipos de unidades denominadas "centro de saúde" C, "centro de saúde" B e "centro de saúde" A, classificados segundo o tipo de programa de saúde pública que lhes cumpre executar. A unidade mais desenvolvida, centro $A$, englohará, portanto, também o programa das menos desenvolvidas, isto é, centros B e C.

Quanto à distribuição geográfica dessas unidades sanitárias, propõe-se que cada unidade sub-regional do Município de São Paulo ou unidade PUB, contenha pelo menos um centro tipo $A$ desde que apresente um limite mínimo de 100.000 habitantes e um máximo de 400.000 a 500.000 habitantes. Acima dêsse valor, até 800.000 habitantes, justifica-se a instalação de 2 centros $\mathrm{A}$ e, finalmente, acima dêste último parâmetro, necessitar-se-á de 3 tipos $\mathrm{A}$ que serão o limite máximo dêsse tipo por unidade de análise.

O padrão proposto do centro de saúde $B$ será de um centro para cada 100.000 habitantes das respectivas unidades de análise adotadas no PUB. Do número de unidades de centro de saúde $B$ necessárias, deverá ser diminuído o número de unidades do padrão $A$, pois êste engloba sempre os serviços prestados pelo padrão $B$.

Para a proposição do padrão $C$ levou-se em consideração, além do tamanho da população, a classe de renda, o raio máximo de atendimento e a densidade.

Para garantir-se um raio de atendimento máximo de $1 \mathrm{~km}$, principalmente para as populações que habitam áreas de baixa densidade, propõe-se um centro de saúde tipo $\mathrm{C}$, para cada 25.000 habitantes em média nos locais onde está previsto predominância de classe social de baixa renda. Do número de unidades do tipo C necessárias, deverá ser diminuído o número de unidades dos padrões mais altos.

A instalação das unidades sanitárias polivalantes deverá obedecer ao seguinte critério: para os centros de menor padräo, ärea construída de 500 a $600 \mathrm{~m}^{2}$ e para os maiores, de 1.100 a $1.400 \mathrm{~m}^{2}$. A área do terreno prevista para as menores unidades deverá ser de $1.500 \mathrm{~m}^{2} \mathrm{e}$, para as maiores, de 2.500 a $3.000 \mathrm{~m}^{2}$.

\section{3 - PROGRAMAS A SEREM \\ DESENVOLVIDOS PELAS UNIDADES SANITARIAS PROPOSTAS}

As diferentes atribuições a serem desen. volvidas por tipo de unidade sanitária proposta são as mesmas previstas pelo decreto-lei n. 50.192 de 13 de agôsto de 1968, pela reforma administrativa da Secretaria de Saúde do Estado.

\section{1 - Centro de Saude C}

Caberá a êste tipo de unidade desenvolver um programa que compreenderá:

a) contrôle de doenças transmissíveis

b) saneamento do meio

c) higiene materno-infanti]

d) assistência médico-sanitária não especializada

e) contrôle da tuberculose e da hanseníase, a cargo de clínico geral

f) epidemiologia e estatística

g) enfermagem

h) educação sanitária

i) administração.

\section{2 - Centro de Saúde B}

Caberá desenvolver programas com. preendendo:

a) contrôle de doenças transmissíveis

b) saneamento do meio

c) higiene materno-infantil

d) assistência médico-sanitária

e) contrôle da tuberculose e da hanseníase, não obrigatòriamente por especialistas 
YUNES, J.; CARDOSO, J. F. \& MENDONÇA, M. L. de - Avaliação das necessidades dos serviços de saúde pública para o munícipio. Rev. Saúde públ., S. Paulo, 3(2):213-217, dez. 1969.

f) odontologia sanitária

g) nutrição

h) epidemiologia e estatística

i) enfermagem

j) educação sanitária

k) laboratório

1) administração.

\section{3 - Centro de Saúde A}

Este seria o mais especializado, cabendo-lhes desenvolver programas que compreendessem :

a) contrôle de doenças transmissiveis

b) saneamento do meio

c) higiene materno-infantil

d) assistência médico-sanitária especializada

e) contrôle de tuberculose e da hanseníase f) odontologia sanitária

g) nutrição

h) epidemiologia e estatística

i) enfermagem

j) educação sanitária

k) laboratório

l) administração.

\section{4 - NECESSIDADES A MÉDIO LONGO PRAZO}

Projetando-se o padrão đefinido anteriormente, necessitaremos para o Município de São Paulo, a médio prazo onde a população prevista é de 7.000.000 de habitantes, um total de 200 unidades polivalentes, das quais 25 serão Centros de Saúde tipo A, 50 do tipo B e, finalmente, 125 do tipo C.

Para o mesmo município, onde a estimativa populacional a longo prazo é da

T A B E L A 1

Município de São Paulo - Necessidades de unidades sanitárias segundo as unidades PUB 1975 e 1990

\begin{tabular}{|c|c|c|c|c|c|c|c|c|}
\hline \multirow{3}{*}{ Unidades PUB } & \multicolumn{8}{|c|}{ Un id a d es s a n i tár i a s } \\
\hline & \multicolumn{4}{|c|}{ Até 1975} & \multicolumn{4}{|c|}{ Até 1990} \\
\hline & Total & $\begin{array}{c}\text { Tipo } \\
\text { A }\end{array}$ & $\begin{array}{c}\text { Tipo } \\
\text { B }\end{array}$ & $\begin{array}{c}\text { Tipo } \\
\text { C }\end{array}$ & Total & Tipo & $\begin{array}{c}\text { Tipo } \\
\text { B }\end{array}$ & $\begin{array}{c}\text { Tipo } \\
\text { C }\end{array}$ \\
\hline Centro & 18 & 2 & 7 & 9 & 23 & 3 & 9 & 11 \\
\hline Lapa & 15 & 2 & 4 & 9 & 18 & 2 & 6 & 10 \\
\hline Casa Verde & 10 & 1 & 7 & 7 & 10 & 1 & 2 & 7 \\
\hline Santana & 12 & 2 & 3 & 7 & 16 & 2 & 5 & 9 \\
\hline Tucuruvi & 7 & 1 & 1 & 5 & 10 & 1 & 2 & 7 \\
\hline Vila Maria & 7 & 1 & 1 & 5 & 7 & 1 & 1 & $\mathbf{5}$ \\
\hline Brás-Moóca & 17 & 2 & 5 & 10 & 18 & 2 & 6 & 10 \\
\hline Vila Prudente & 11 & 1 & 2 & 8 & 13 & 1 & 3 & 9 \\
\hline Ipiranga & 7 & 1 & 2 & 4 & 9 & 1 & 3 & 5 \\
\hline Saúde & 9 & 1 & 3 & 5 & 11 & 1 & 4 & 6 \\
\hline Ibirapuera & 8 & 1 & $\mathbf{3}$ & 4 & 10 & 1 & 4 & 5 \\
\hline Pinheiros & 8 & 1 & 3 & 4 & 12 & 2 & 4 & 6 \\
\hline Belezas & 5 & 1 & 1 & 3 & 9 & 1 & 2 & 6 \\
\hline Santo Amaró & 7 & 1 & 2 & 4 & 10 & 1 & 4 & 5 \\
\hline Penha & 21 & 2 & 4 & 15 & 23 & 2 & $\mathbf{5}$ & 16 \\
\hline Itaquera & 8 & 1 & 1 & 6 & 13 & 1 & 3 & 9 \\
\hline São Miguel & 6 & 1 & 1 & 4 & 7 & 1 & 1 & 5 \\
\hline Guaianazes & 6 & 1 & 1 & 4 & 8 & 1 & 2 & 5 \\
\hline São Matheus & 7 & 1 & 1 & 5 & 16 & 1 & 4 & 11 \\
\hline São Paulo Sul & 9 & 1 & 2 & 6 & 31 & 2 & 7 & 22 \\
\hline Perús & 2 & - & 1 & 1 & 6 & - & 2 & 4 \\
\hline Total & 200 & 25 & 50 & 125 & 280 & 28 & 79 & 173 \\
\hline
\end{tabular}


YUNES, J.; CARDOSO, J. F. \& MENDONÇA, M. L. de - Avaliação das necessidades dos serviços de saúde pública para o municipio. Rev. Saúde púbi., S. Paulo, 3(2):213-217, dez. 1969.

ordem de 10.000.000 de habitantes, as necessidades previstas serão de 280 unidades sanitárias integradas, das quais 28 serão Centros de Saúde tipo A, 79 do tipo B e 173 do tipo C.

A Tabela 1 resume estas necessidades projetadas a curto e a longo prazo.

Adotou-se, como critério geral, que quando o número de unidades sanitárias necessárias para atender ao padrão proposto fôr maior do que o número de unidades atualmente existentes, serão construídas novas unidades. Exceção feita à unidade PUB denominada Centro e Brás. -Moóca, onde a densidade populacional é maior e os locais disponíveis para novos Centros de Saúde pràticamente inexistem. Havendo "excesso" de unidades sanitárias por unidade PUB, adotou-se, como critério, adequar os atuais Centros de Saúde: em tipo A, os Postos de Saúde em tipo B e os Sub-Centros ou Postos de Higiene Materno-Infantil em tipo $\mathrm{C}$, até completar as necessidades propostas a médio e longo prazo.

De acôrdo com o crilério acima expos. to, resumimos na Tabela 2 as necessidades de unidades sanitárias a construir e a adequar a médio e a longo prazo e comparamos com a situação atual existente. Para 1975 será necessário construir 59 unidades sanitárias, das quais 9 serão do tipo $\mathrm{A}, 24$ do tipo $\mathrm{B}$ e 26 do tipo C. O número de unidades sanitárias necessárias a adequar para o mesmo ano, será de 141 , sendo 16 para o tipo A, 26 para o tipo B e 99 para o tipo C. Para o ano de 1990, o número de unidades necessárias a construir será de 123 , sendo 10 Centros de Saúde do tipo $A, 50$ do tipo $B$ e 63 do tipo C. Para o mesmo ano, necessitar-se-á adequar 157 unidades, das quais 18 para Centro de Saúde $A, 29$ para B e 110 para C.

\section{5 - RESPONSABILIDADE FUNCIONAL}

A participação da Prefeitura nos Serviços Oficiais de Saúde Pública será, fundamentalmente, de prover os Postos de Saúde tipo $\mathrm{C}$ e os novos postos do tipo $\mathrm{B}$.

A responsabilidade funcional do poder público municipal e estadual deverá objetivar como principal clientela a população indigente.

Ao Instituto Nacional de Previdência Social caberá todo o atendimento médico à população previdenciária e seus dependentes.

Ao Estado caberá enfatizar a sua ação em Medicina Preventiva e Saúde Pública, continuando a sua participação nas unidades oficiais de Saúde Pública, principalmente nos Centros de Saúde tipo B e A, que compreenderão o atendimento especializado.

T A B E L A 2

Municipio de São Paulo - Necessidades de unidades sanitárłas a construir e adequar a médio e a longo prazo - 1975 e 1990

\begin{tabular}{|c|c|c|c|c|c|c|c|}
\hline \multirow{2}{*}{ Discriminação } & \multirow{2}{*}{$\begin{array}{l}\text { Situa- } \\
\text { cão } \\
\text { atual }\end{array}$} & \multicolumn{2}{|c|}{ Necessidades } & \multicolumn{2}{|c|}{$\begin{array}{c}\text { A construir e } \\
\text { equipar }\end{array}$} & \multicolumn{2}{|c|}{ A adequar } \\
\hline & & $\begin{array}{l}\text { Até } \\
1975\end{array}$ & $\begin{array}{c}\text { Até } \\
1990\end{array}$ & $\begin{array}{l}\text { Até } \\
1975\end{array}$ & $\begin{array}{l}\text { Até } \\
1990\end{array}$ & $\begin{array}{l}\text { Até } \\
1975\end{array}$ & $\begin{array}{l}\text { Até } \\
1990\end{array}$ \\
\hline Unidades Sanitárias: & 193 & 200 & 280 & 59 & 123 & 141 & 157 \\
\hline Centro de Saúde Tipo & 159 & 125 & 173 & 26 & 63 & 99 & 110 \\
\hline Centro de Saúde tipo & 12 & 50 & 79 & 24 & 50 & 26 & 29 \\
\hline Centro de Saúde Típo & 22 & 25 & 28 & 9 & 10 & 16 & 18 \\
\hline
\end{tabular}

Nota: Dos Postos de Puericultura a adequar para o Centro C, 38 pertencem à Prefeitura. 
YUNES, J.; CARDOSO, J. F. \& MENDONÇA, M. L. de - Avaliação das necessidades dos serviços de saúde pública para o município. Rev. Saúde públ., S. Paulo, 3(2):213-217, dez. 1969.

Yunes, J.; Cardoso, J. F. \& Mendonça, M. L. de - Estimate of needs in public health official services for the City of São Paulo at short and long run. Rev. Saúde puibl., S. Paulo, 3(2): 213-217, dez. 1969 .

Summary - This paper refers to the demand of sanitary unities, at short and long run, for the years of 1975 and 1990, respectively. This study was part of the "Plano Urbanístico Básico de São Paulo (PUB)", and the proposition always followed the criterium of integrated or polivalent unities. In the definition of the proposed patterns it was considered the size of population, demographic density, size and extension of the area and socio-economic classes. The sanitary unities were divided into three types, and their programs established according to their purposes. The "municipal" authorities are responsible for their functioning, as well as the state and welfare agencies. 